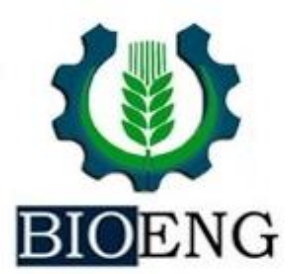

\section{ANÁLISE DA PERTURBAÇÃO ACÚSTICA PRODUZIDA POR MOTOSSERRAS E ROÇADORAS EM DIFERENTES AMBIENTES}

\author{
L. M. Santos ${ }^{1 *}$, G. A. e S. Ferraz ${ }^{1}$, F. B. D. S. Martins ${ }^{2}$, \\ R. R. Salvador ${ }^{1}$, P. F. P. Ferraz ${ }^{1}$
}

${ }^{1}$ UFLA - Universidade Federal de Lavras, Departamento de Engenharia, Lavras, MG, Brasil
${ }^{2}$ UFRRJ - Universidade Federal Rural do Rio de Janeiro, Departamento de Engenharia,
Seropédica, RJ, Brasil

Article history: Received 08 March 2019; Received in revised form 03 May 2019; Accepted 06 May 2019; Available online 24 June 2019.

\title{
RESUMO
}

O setor florestal sofreu grande avanço com a introdução das motosserras e roçadoras em seu processo. Apresentando um custo relativamente baixo e facilidade de operação, as máquinas portáteis tornaram-se essenciais. O ruído está presente na rotina diária dos seres humanos e, esse complexo de sons, pode causar sensações de desconforto. O presente trabalho teve como objetivo analisar o nível de ruídos emitido por três modelos de motosserras e dois modelos de roçadoras. $\mathrm{O}$ experimento foi realizado em três ambientes: dentro de uma oficina, dentro de uma cabine de testes e no campo (ambiente de trabalho), testando-se três motosserras diferentes e duas roçadoras a combustão e uma à bateria, sendo operadas em duas rotações (baixa e alta). Para a mensuração do ruído emitido por estas máquinas foi utilizado um decibelímetro. Os dados foram analisados por meio de agrupamento hierárquico aglomerativo. Observou-se que a potência da máquina, a rotação e o ambiente foram preponderantes para o ruído emitido por estas máquinas, além disso, todas as motosserras testadas apresentaram ruídos muito superiores a $85 \mathrm{~dB}(\mathrm{~A})$, preconizados pela norma, quando operados na oficina e em ambiente de trabalho e para as duas rotações testadas. Operadas dentro da cabine de testes, os motosserras apresentaram-se dentro dos padrões da norma, independentemente da rotação. Já a roçadora a combustão apresentou valores superiores a $85 \mathrm{~dB}$ (A), para as duas rotações avaliadas quando operada em campo aberto, enquanto que a roçadora a bateria apresentou valores superiores a $85 \mathrm{~dB}(\mathrm{~A})$ apenas para alta rotação.

Palavras-chave: Ruído ocupacional, segurança no trabalho, máquinas portáteis.

\section{ANALYSIS OF ACOUSTIC PERTURBATION PRODUCED BY CHAINSAW AND BRUSHCUTTER IN DIFFERENT ENVIRONMENTS}

\begin{abstract}
The forestry sector has made great progress with the introduction of chainsaws and brushcutters in its process. Featuring a relatively low cost and ease of operation portable machines have become essential. Noise is present in the daily routine of humans; this complex of sounds can cause feelings of discomfort. The present work had as objective to analyze the noise level emitted by three models of chainsaws and two models of brushcutter. The experiment was carried out in three environments, inside a workshop, inside a test booth and in the field (work environment), being tested three different chainsaws and two burners
\end{abstract}

\footnotetext{
*luanna_mendess@yahoo.com.br
} 
and one to the battery, being operated in two rotations (low and high). A decibel meter was used to measure the noise emitted by these machines. Data were analyzed by agglomerative hierarchical clustering. It was observed that the power of the machine, the rotation and the environment were preponderant for the noise emitted by these machines, in addition all the chainsaws tested showed noises much superior to $85 \mathrm{db}$ (A), recommended by the standard, when operated in the workshop and in working environment and for the two rotations tested. Operated within the test cabin, the chainsaws were within the standards of the standard, regardless of rotation. On the other hand, the burner had values higher than $85 \mathrm{~dB}(\mathrm{~A})$, for the two rotations evaluated when operated in the open field, while the battery had values higher than $85 \mathrm{~dB}(\mathrm{~A})$ only for high rotation.

Keywords: Occupational Noise, workplace safety, portable machines.

\section{INTRODUÇÃO}

Segundo IBGE (2015) a crescente produção da extração vegetal e da silvicultura acarretou no ano de 2016 uma soma de R\$ 18,4 bilhões. A silvicultura (obtida em florestas plantadas) contribuiu com 74,3\% (R \$ 13,7 bilhões) do total, enquanto a extração vegetal (coleta ou apanha de produtos em matas e florestas nativas) participou com 25,7\% (R\$ 4,7 bilhões). $\mathrm{O}$ corte florestal semimecanizado com motosserra é o mais difundido no Brasil, além da limpeza do terreno que utilizam as roçadoras.

Diante do cenário de crescimento neste setor, os trabalhadores nem sempre têm condições favoráveis de trabalho. A Colheita Florestal, de acordo com Fonseca et al. (2017), faz uso de muitos equipamentos que prejudicam as capacidades auditivas dos operadores por produzirem altos níveis de ruído. Atualmente, tem-se aumentado o número de empreiteiras na indústria florestal, aumentando a exploração de madeiras usando trituradores e máquinas ruidosas expondo os trabalhadores a longas horas de jornada, sendo uma situação preocupante em relação à exposição do trabalhador ao ruído (POJE et al., 2015).

Visto que o ruído é um dos principais agentes acústicos no setor florestal, a Associação Brasileira de Normas Técnicas (ABNT) possui algumas normas sobre medições de ruído em máquinas do setor, sendo as principais a NBR 9999 (ABNT, 1987a) e a NBR 10400 (ABNT, 1988). A
Norma NB 95 (ABNT, 1987b) estabelece os níveis máximos de ruído que permitem o mínimo de conforto aos ocupantes de um ambiente. O máximo estabelecido é de 85 $\mathrm{dB}(\mathrm{A})$, e acima desse limite o ruído, além de perturbar as atividades humanas, pode causar danos à audição.

Sabe-se, também, que o som é a variação da pressão ambiente detectável pelo sistema auditivo e ruído é um som sem harmonia, em geral de conotação negativa, ou seja, que na maioria das vezes pode ser classificado como um som indesejável (BISTAFA, 2006; DAMASCENO et al., 2009). Diante disso, pode-se perceber que diferente de outros tipos de poluição, a poluição sonora não deixa traços visíveis de sua influência no ambiente. Essa é, depois da poluição do ar e da água, o problema ambiental que afeta o maior número de pessoas (NASCIMENTO et al., 2007).

Segundo a Norma Regulamentadora (NR) $n^{\circ} 15$ anexo I da Portaria 3214/78 do Ministério do Trabalho e Emprego, atualizada em 2014 (BRASIL, 2018c) define ruído contínuo ou intermitente, para os fins de aplicação de limites de tolerância, o ruído que não seja ruído de impacto. Tal NR estabelece que, para operadores que não estejam protegidos adequadamente, não é permitido a exposição a níveis de ruído acima de 115 $\mathrm{dB}(\mathrm{A})$.

A exposição ocupacional ao ruído em níveis elevados, pode provocar uma 
série de efeitos colaterais segundo Fonseca et al. (2017), danificando os ouvidos, podendo ocasionar danos irreversíveis à audição e comprometer a qualidade de vida. Em seus estudos, Araújo et al. (2013) consideram que o perfil das doenças dos trabalhadores florestais é um desafio clínico, pois, geralmente, é resultado da junção de diversos fatores, o que dificulta seu diagnóstico e tratamento.

Para garantir um ambiente salubre em setores que envolvem trabalhos na agricultura, pecuária silvicultura, exploração florestal e aquicultura, a NR 31 (BRASIL, 2018d) estabelece que o empregador rural ou equiparado, de acordo com as necessidades de cada atividade, deve fornecer aos trabalhadores protetores auriculares para as atividades com níveis de ruído prejudiciais à saúde. Visto que o setor florestal, em grande parte, dispõe de

\section{MATERIAL E MÉTODOS}

O trabalho foi realizado no Departamento de Engenharia da Universidade Federal de Lavras DEG/UFLA - Lavras, MG. Foram avaliadas roçadoras à combustão e à bateria e motosserras com potências e ambientes diferentes (Tabela 1).

Foram avaliados os níveis de ruído emitido pelas roçadoras e pelas motosserras, registrando-os quando as máquinas foram operadas em baixa rotação atividades árduas e desgastantes, em alguns casos, trabalhos precários e sem condições mínimas de segurança, estudo de riscos inerentes a atividade deste setor se faz necessário para eliminá-los ou reduzi-los.

Assim conhecer os níveis de ruídos de máquinas e realizar um levantamento dos riscos que danificam a saúde dos operadores. E, consequentemente, propor medidas mitigadoras que atenuam tais riscos. Com isso, o presente trabalho teve como objetivo realizar um levantamento dos níveis de ruídos medidos com o uso de um decibelímetro digital modelo dec 460, por três modelos de motosserras e dois tipos de roçadoras portáteis em diferentes ambientes de trabalho, analisando os dados através de agrupamento hierárquico aglomerativo, a fim de comparar com os valores estipulados pela NR 15 .

e em alta rotação. Além das diferentes rotações, os ruídos foram mensurados, também, dentro de uma cabine de testes, em ambiente externo e as motosserras foram avaliadas no interior de uma oficina, esta situação foi considerada devido às manutenções necessárias que tais máquinas precisam passar dentro da oficina. Para cada tratamento foram realizadas 15 (quinze) repetições.

Tabela 1. Especificações técnicas das motosserras e roçadoras utilizadas.

\begin{tabular}{|c|c|c|c|c|c|}
\hline & $\begin{array}{c}\text { Potência } \\
(\mathrm{kW})\end{array}$ & $\begin{array}{c}\text { Cilindrada } \\
\left(\mathrm{cm}^{3}\right)\end{array}$ & $\begin{array}{c}\text { Rotação } \\
\text { máxima }(\mathrm{rpm})\end{array}$ & $\begin{array}{c}\text { Rotação } \\
\text { Mínima }(\mathrm{rpm})\end{array}$ & $\begin{array}{c}\text { Peso } \\
(\mathrm{kg})\end{array}$ \\
\hline $\begin{array}{c}\text { Roçadora à } \\
\text { combustão }\end{array}$ & 1,6 & 40,2 & 12.300 & 2.800 & 7,3 \\
\hline Roçadora à bateria & 1,2 & - & 6.500 & 3.500 & 4,5 \\
\hline $\begin{array}{c}\text { Motosserra A à } \\
\text { combustão }\end{array}$ & 1,3 & 30,1 & 14.000 & 2.800 & 3,9 \\
\hline $\begin{array}{c}\text { Motosserra B à } \\
\text { combustão }\end{array}$ & 2,6 & 50,2 & 14.000 & 2.800 & 4,8 \\
\hline $\begin{array}{c}\text { Motosserra C à } \\
\text { combustão }\end{array}$ & 3,9 & 72,2 & 12.500 & 2.400 & 6,6 \\
\hline
\end{tabular}

Os dados foram mensurados por meio de um decibelímetro digital modelo dec 460, operando no circuito de resposta lenta (Slow) e de equalização "A", 
conforme o anexo 1 da NR 15 (BRASIL, 2018c), assim como o protetor de vento do respectivo medidor foi utilizado em todas as medições.

Os níveis de ruído avaliados em uma cabine de teste, cujas paredes são revestidas de modo a evitar entrada de ruídos externos, foram medidos em seu centro, na altura média do ouvido do operador. Em ambiente externo, a mensuração também se deu próximo à altura média do ouvido do operador e distante de paredes e anteparos assim como na oficina.

\section{RESULTADOS E DISCUSSÃO}

Observa-se na Tabela 2 os valores médios de ruídos emitidos pelas motosserras e pelas roçadoras avaliadas quando operadas em alta e baixa rotação, dentro de uma câmara de testes, em ambiente externo e na oficina (somente as motosserras). Nesta tabela pode-se observar, também, que de acordo com os dados da NR 15 (BRASIL, 2018c), quando se opera a motosserra A em ambiente externo na rotação máxima, o tempo máximo limite de exposição ao ruído emitido gerado por ela é de 20 minutos, enquanto que na oficina em alta rotação os níveis extrapolaram o valor máximo permitido pela norma e em baixa rotação na oficina o máximo permitido seria de 1 hora. Quando se opera a motosserra B em ambiente externo e na máxima rotação, o tempo máximo limite de exposição é de 7 minutos e na oficina seguem os mesmos valores que os da motosserra A. Já quando se opera motosserra $\mathrm{C}$ nas mesmas condições descritas para as motosserras A e $\mathrm{B}$, observa-se que o limite máximo de exposição ao ruído é de 8 minutos. Observa-se ainda nesta tabela, que apenas
Os resultados foram analisados com base nos limites de tolerância para ruídos contínuos, estabelecidos pela Legislação Brasileira constados na NR 15 (BRASIL, 2018c) e analisados através de agrupamento hierárquico aglomerativo.

As Análises de Agrupamento Hierárquico Aglomerativo (HACA) e a confecção dos gráficos de dendrogramas das médias de ruídos emitidos pelas motosserras e pelas roçadoras nas diferentes rotações e nos diferentes ambientes foram realizadas utilizando $\mathrm{O}$ sistema computacional estatístico R (R Development Core Team, 2017).

dentro da câmara de testes é que os valores do ruído das motosserras em estudo, permaneceram abaixo de $85 \mathrm{~dB}(\mathrm{~A})$, onde a NR 15 (BRASIL, 2018c) preconiza que o operador pode ficar exposto por 8 horas sem uso de equipamentos de proteção individual (EPI).

No caso das roçadoras, quando o operador utiliza a roçadora à combustão em alta rotação o nível observado, permitiria, segundo a NR 15, que este ficasse exposto por no máximo 15 minutos em ambiente externo e na câmara de testes poderia atuar por 8 horas. Para a roçadora a bateria os níveis em ambiente externo propõe uma carga de 1 hora e 45 minutos de trabalho e na câmara de testes obteve valor muito inferior ao mínimo que a NR 15 (BRASIL, 2018c) propõe, obedecendo aos mesmos conselhos para a operação da roçadora a combustão em câmera de teste. 
Tabela 2 - Valores médios de ruído $(\mathrm{dB}(\mathrm{A}))$ emitido pelas motosserras e pelas roçadoras em diferentes rotações e em diferentes locais de avaliação.

\begin{tabular}{ccccccc}
\hline & \multicolumn{2}{c}{ Oficina } & \multicolumn{2}{c}{ Câmara de Testes } & \multicolumn{2}{c}{ Ambiente Externo } \\
\hline & Baixa & Alta & Baixa & Alta & Baixa & Alta \\
Rotação & Rotação & Rotação & Rotação & Rotação & Rotação \\
\hline A & 99,9 & 116,3 & 66,6 & 73,5 & 92,8 & 107,9 \\
B & 94,0 & 117,0 & 66,6 & 71,8 & 98,3 & 115,6 \\
C & 101,2 & 122,2 & 69,7 & 79,9 & 94,4 & 114,0 \\
Roçadora à & & & & & & \\
combustão & - & - & 73,2 & 80,5 & 89,3 & 109,3 \\
Roçadora à bateria & - & - & 69,6 & 73,3 & 76,9 & 95,6 \\
\hline
\end{tabular}

Nota-se que os níveis de ruídos foram maiores em alta rotação e dentro da oficina, de acordo com os estudos realizados por Niemeyer \& Santos (2001), este resultado era de se esperar uma vez que o som dentro de espaços fechados sofre diversas reflexões, diferente do espaço aberto em que a atenuação ocorre na medida em que se afasta da fonte emissora, dentro do espaço fechado, a atenuação ocorre pela perda de energia devido às reflexões do som, os autores ainda afirmam que dentro do espaço fechado o nível de ruído é superior ao do espaço aberto por ser um somatório de som direto com reflexões múltiplas. Estes resultados são importantes para as medidas de controle e atenuação que devem ser tomadas para que os trabalhadores envolvidos nesta situação não venham a sofrer por Perda Auditiva Induzida por Ruído Ocupacional (PAIRO).

A PAIRO é considerada a perda auditiva ocorrida no ambiente de trabalho, que progride com o tempo de exposição e tem como característica ser irreversível, com alguns sintomas como: zumbido, dificuldade no entendimento da fala, sensação de plenitude auricular e sensação de audição "abafada", bem como o recrutamento, presente em praticamente todos os casos (MENIN et al., 2014).

Para efeitos de cumprimento de legislação, a situação de uma possível manutenção de tais máquinas (motosserra A, B e C) dentro da oficina estaria em não conformidade com a legislação vigente, ou seja, acima do limite de tolerância estabelecido pela NR 15 (BRASIL, 2018c), logo os trabalhadores estarão em situação insalubre podendo estes receberem por tais danos. Neste caso, as medidas hierárquicas de controle, segundo a NR 9 (BRASIL, 2018b) que trata do Programa de Prevenção de Riscos Ambientais, prevê que sejam adotadas medidas de proteção coletiva como a substituição ou modificação da máquina; medidas de caráter administrativo ou de organização do trabalho como enclausuramento da máquina, diminuição do tempo de trabalho utilizando estas máquinas ou isolamento acústico da oficina e por fim, se as medidas acima não forem suficientes ou até que as ações sejam tomadas, a utilização de equipamento de proteção individual adequado que atenue os níveis de ruído encontrados.

No caso da utilização tanto da roçadora quanto das motosserras em ambientes externos, as medidas que devem ser tomadas é a substituição de máquinas por outras que produzam ruídos dentro dos limites permitidos pela legislação vigente, caso não tenha esta possibilidade, o empregador deverá adotar medidas de proteção individual com o uso de protetores auriculares que atenuem os níveis de ruídos que os trabalhadores estão expostos, como é estabelecido na NR 31 (BRASIL, 2018d) e na NR 6 que trata de equipamentos de proteção individual (EPI) (BRASIL, 2018a).

$\mathrm{Na}$ Figura 1 pode-se observar a análise de agrupamento, em que a motosserras $\mathrm{A}, \mathrm{B}$ e $\mathrm{C}$ em baixa rotação dentro da oficina apresentaram ruídos estatisticamente iguais ao ruído emitido 
quando operando em baixa rotação. Já no ambiente externo devido ao nível de exposição a ruídos do meio ambiente apresentando um tempo limite de exposição na oficina em torno de 1 hora, enquanto que no ambiente externo entre 2 e 3 horas de exposição. Assim que operadas em alta rotação comparando estes dois ambientes houve uma leve variação devido à potência e cilindrada de cada motosserra, sendo a motosserra $\mathrm{C}$ a mais ruidosa. Quando operadas na cabine de testes os valores de níveis de ruído foram estatisticamente iguais devido a composição de proteção da cabine.

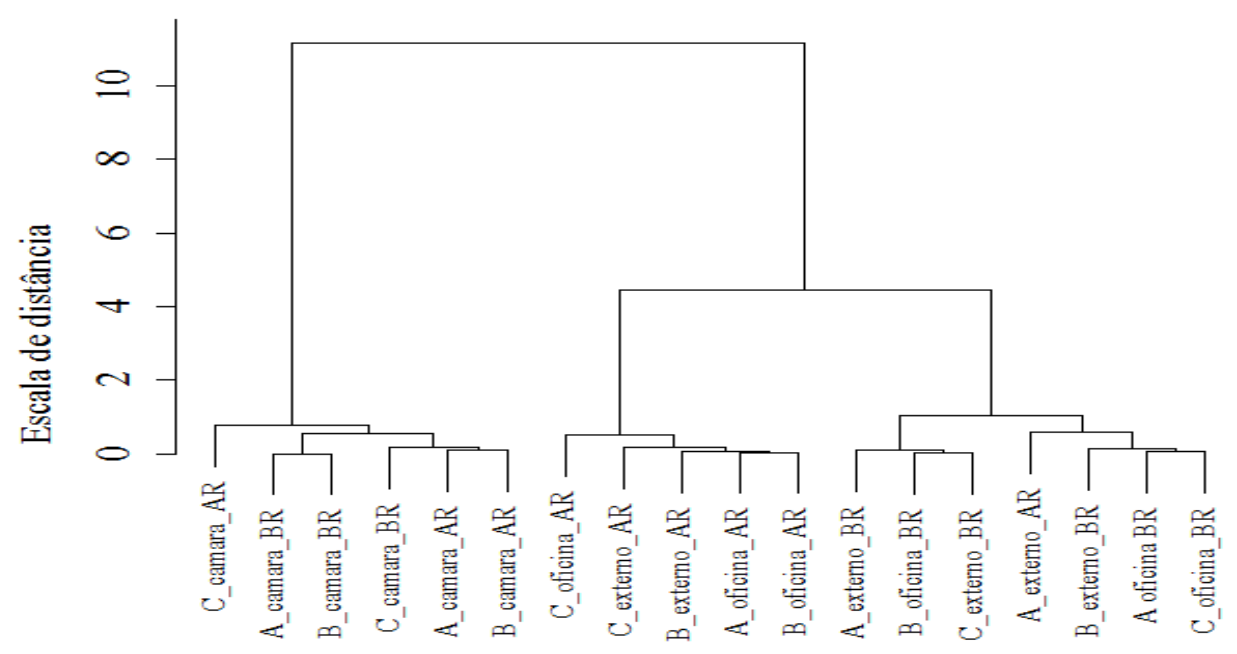

Figura 1. Dendrograma do ruído emitido pelas motosserras A, B e C, na câmara de testes (câmara), no interior da oficina (oficina), no ambiente externo (externo) em alta rotação (AR) e baixa rotação $(\mathrm{BR})$.

$\mathrm{Na}$ Figura 2 pode-se observar o agrupamento, em que a roçadora a combustão operando em baixa rotação dentro da câmara de testes apresentou um ruído estatisticamente igual ao ruído emitido pela roçadora a bateria operando em alta rotação em câmara de teste devido ao nível de proteção da câmara. Assim como a roçadora a combustão atuando em alta rotação na câmara de teste apresentou valores estatisticamente próximos ao da roçadora a bateria em baixa rotação atuando no ambiente externo, devido ao fator ambiental atuando na roçadora a bateria, aumento os níveis de ruído e em relação a roçadora a combustão o alto índice de proteção que a cabine dispõe fez os valores ficarem próximos. 


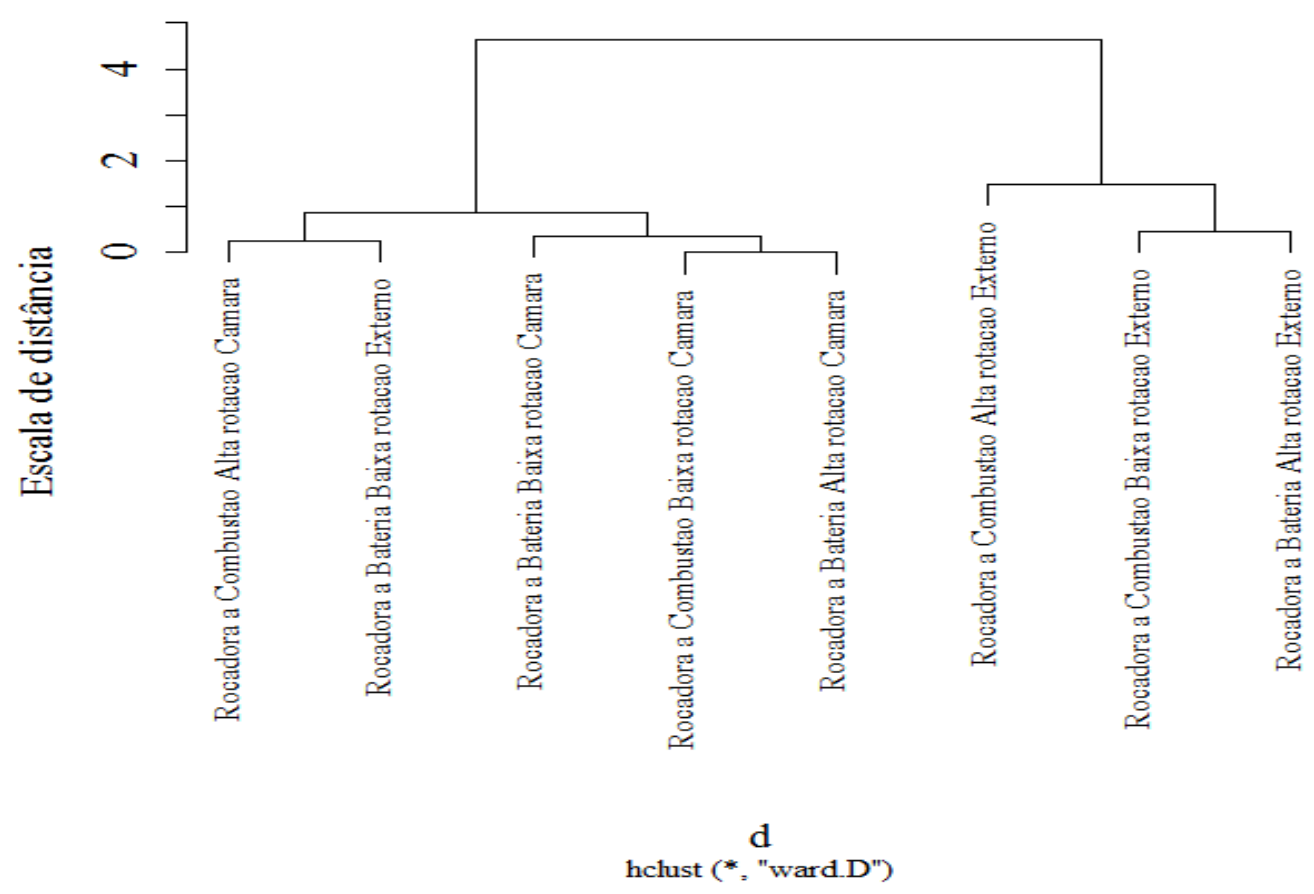

Figura 2. Dendrograma do ruído emitido pelas roçadoras durante o período experimental na câmara de testes (câmara), no ambiente externo (externo) em alta rotação (AR) e baixa rotação (BR).

Em estudo realizado com um grupo de trabalhadores florestais para avaliar as condições da exposição ocupacional ao ruído emitido por trator, motosserras e roçadora, Fonseca et al. (2017) observaram que em todas as avaliações foi excedido valor de $85 \mathrm{~dB}(\mathrm{~A})$ permitido para jornada de trabalho de $8 \mathrm{~h}$, além disso concluíram que operadores de motosserras apresentaram o maior nível de exposição ao ruído entre as três ocupações analisadas, sendo o caso mais crítico, pois alcançou o Limite de Exposição Valor Teto (LE-VT) de $115 \mathrm{~dB}(\mathrm{~A})$, ou seja, o valor de máxima exposição permitida na NR 15 (BRASIL, 2018c) para exposição ao ruído continuo e intermitente, sendo considerado uma

\section{CONCLUSÕES}

Foi possível realizar o levantamento dos níveis de ruídos emitido pelas motosserras e roçadoras avaliadas e analisar de acordo com os valores estabelecidos pela NR 15 por meio de agrupamentos hierárquico aglomerativo.

Das motosserras avaliadas, a que mais expôs o trabalhador aos riscos de situação de risco grave e eminente passível de interdição.

Assim, verifica-se que estudos quantificando a exposição ao risco são importantes para tomar medidas que minimizem a exposição dos trabalhadores a agentes insalubres no ambiente de trabalho, além de evitar acidentes e doenças ocupacionais. Pio da Silva et al. (2009) alertam para o fato de que os acidentes e as doenças relacionadas ao trabalho podem impactar não apenas na vida do indivíduo, mas também na sociedade como um todo. Ressalta ainda que esses eventos podem afetar o custo de produção para as empresas, forçando a elevação do preço do produto final, além de prejudicar o desempenho da empresa.

ruídos foi a motosserra $\mathrm{C}$ no ambiente da oficina. Ao comparar os níveis de ruído gerado pelas máquinas avaliadas com o que define a NR 15 pode-se perceber que as motosserras avaliadas emitem ruídos consideráveis para a saúde do operador, tendo a motosserra $\mathrm{B}$ atingido o valor máximo permitido pela NR 15 quando 
operada em ambiente externo. Das roçadoras avaliadas a que mais expõe o trabalhador aos riscos de ruídos foi a máquina a combustão, sendo a mais ruidosa.

Os resultados obtidos no trabalho reforçam a necessidade de medidas de proteção coletiva como a substituição ou

\section{AGRADECIMENTOS}

Os autores agradecem ao Conselho Nacional de Desenvolvimento Científico e Tecnológico (CNPq), à Coordenação de Aperfeiçoamento de Pessoal de Nível Superior (CAPES) e Fundação de Amparo

\section{REFERÊNCIAS}

ARAÚJO, J. N. G.; GREGGIO, M. R.; PINHEIRO, T. M. M. Agrotóxicos: a semente plantada no corpo e na mente dos trabalhadores rurais. Psicologia em Revista, v. 19, n. 3, p. 389-406, 2013.

ABNT- Associação Brasileira De Normas Técnicas. NBR 9999: Medição do Nível de Ruído, no Posto de Operação de tratores e Máquinas Agrícolas. Rio de Janeiro, 1987a.

ABNT- Associação Brasileira De Normas Técnicas. NBR 10152 (NB 95): Níveis de ruído aceitáveis. Rio de Janeiro, 1987b.

ABNT- Associação Brasileira De Normas Técnicas. NBR 10400: Tratores Agrícolas: Determinação das Características Técnicas e Desempenho. Rio de Janeiro, 1988.

BISTAFA, S.R. Acústica aplicada ao controle do ruído. Edgard Blucher. 368p, 2006.

BRASIL. Ministério do Trabalho e Emprego. 2018. Disponível em: <http://trabalho.gov.br/portal-mte/>. Acesso em: 20 maio de 2018. . NR 6 - Equipamentos de Proteção Individual-EPI. Disponível em: < modificação da máquina; medidas de caráter administrativo ou de organização do trabalho como enclausuramento da máquina, diminuição do tempo de trabalho utilizando estas máquinas ou isolamento acústico, no caso da oficina e, por fim, uso de protetores auriculares adequados durante a operação.

à Pesquisa do Estado de Minas Gerais (FAPEMIG) pelo auxilio destinado na realização deste trabalho e ao PET/SISU/MEC.

http://trabalho.gov.br/images/Documentos/ SST/NR/NR6.pdf >. Acesso em: 20 maio 2018a.

NR 9 - Programa de Prevenção de Riscos Ambientais Disponível em: < http://trabalho.gov.br/images/Documentos/ SST/NR/NR09/NR-09-2016.pdf > . Acesso em: 20 maio 2018 b.

$\begin{array}{cc}\text {. NR } 15 \text { - Atividades e Operações } \\ \text { Insalubres. } & \text { Disponível }\end{array}$ <http://trabalho.gov.br/images/Documento s/SST/NR/NR15/NR-15.pdf >. Acesso em: 20 maio $2018 \mathrm{c}$.

NR 31 - Segurança e saúde no
trabalho na agricultura, pecuária
silvicultura, exploração florestal e
aquicultura. $\quad$ Disponível em:
<http://trabalho.gov.br/images/Documento
s/SST/NR/NR31.pdf >. Acesso em: 20
maio 2018d.

DAMASCENO, F.A.; JUNIOR, T.Y.; GOMES, R.C.C.; LIMA, R.R.; SCHIASSI, L.; MORAES, S.R.P. Avaliação do nível de ruído produzido por caminhões de ração no município de Itaberaí (GO). Revista Ciências do Ambiente On-Line, v.4, n.1, p.44-48, 2009. 
FONSECA, A.F.C.; SANTOS, F.D.R.; CATAI, R.E.; AMARILLA, R.S.D. Análise da exposição ocupacional ao ruído em trabalhadores de uma empresa florestal. Espacius, v.38, n.26, p.25-35, 2017.

IBGE - Instituto Brasileiro de Geografia e Estatística (2015) Produção da Extração Vegetal e da Silvicultura-PEVS. Disponível em $<$ https://www.ibge.gov.br/estatisticasnovoportal/economicas/agricultura-epecuaria/9105-producao-da-extracaovegetal-e-dasilvicultura.html $?=\& \mathrm{t}=$ resultados $>$. Acesso em 26 ago. 2017.

MENIN, E.G; KUNZ, B. T.; BRAMATTI, L. Relação da perda auditiva induzida por ruído e o uso de tabaco em trabalhadores de uma indústria alimentícia. Revista CEFAC, v.16, n. 2, p. 384-394, 2014.

NASCIMENTO, R.G.D; GODOY, R.M.B.D.; JUNIOR, C.A.S.; UEHARA, G.T. Avaliação da poluição sonora na Unicamp. Revista Ciências do Ambiente On-Line, v. 3, n. 1, p.1-7, 2007.

NIEMEYER, M.L.; SANTOS, M.J.D.O. Qualidade acústica no espaço urbano. VI Encontro Nacional Sobre O Conforto No Ambiente Construído, São Pedro, 2001.

POJE, A.; SPINELLI, R.; MAGAGNOTTI, N.; MIHELIC, M. Exposure to noise in wood chipping operations under the conditions of agroforestry. International Journal of Industrial Ergonomics, v.50, n.1, p.151157, 2015.

R Development Core Team. R: a language and environment for statistical computing. Vienna: $\mathrm{R}$ Foundation for Statistical Computing. Disponível em: <http://www.R-project.org/>. Acesso em: 15 out. 2017.

PIO DA SILVA, E.; MINETTE, L.J.; SOUZA, A.P.D.; COSTA BAÊTA, F.D.;
FERNANDES, H.C.; MAFRA, S. C. T.; VIEIRA, H.A.N.F. Caracterização da saúde de trabalhadores florestais envolvidos na extração de madeira em regiões montanhosas. Revista Árvore, v.33, n.6, p.1169-1174, 2009. 